\title{
On Killing Forms and Invariant Forms of Lie-Yamaguti Superalgebras
}

\author{
Patricia L. Zoungrana ${ }^{1,2}$ and A. Nourou Issa ${ }^{1,2}$ \\ ${ }^{1}$ Département de Mathématiques de la Décision, Université Ouaga 2, 12 BP 412, Ouagadougou 12, Burkina Faso \\ ${ }^{2}$ Département de Mathématiques, Université d’Abomey-Calavi, 01 BP 4521, Cotonou, Benin \\ Correspondence should be addressed to Patricia L. Zoungrana; patibffr@yahoo.fr
}

Received 15 October 2016; Accepted 20 December 2016; Published 12 January 2017

Academic Editor: Hernando Quevedo

Copyright (c) 2017 P. L. Zoungrana and A. N. Issa. This is an open access article distributed under the Creative Commons Attribution License, which permits unrestricted use, distribution, and reproduction in any medium, provided the original work is properly cited.

\begin{abstract}
The notions of the Killing form and invariant form in Lie algebras are extended to the ones in Lie-Yamaguti superalgebras and some of their properties are investigated. These notions are also $\mathbb{Z}_{2}$-graded generalizations of the ones in Lie-Yamaguti algebras.
\end{abstract}

\section{Introduction}

A Lie-Yamaguti algebra is a triple $(T, *,[\cdot, \cdot, \cdot])$ consisting of a vector space $T$, a bilinear map $*: T \times T \rightarrow T$, and a trilinear map $[\cdot, \cdot, \cdot \cdot]: T \times T \times T \rightarrow T$ such that

$$
\begin{aligned}
& \text { (LY1) } x * y=-y * x, \\
& (\mathrm{LY} 2)[x, y, z]=-[y, x, z], \\
& (\mathrm{LY} 3) \sigma_{x, y, z}\{((x * y) * z)+[x, y, z]\}=0, \\
& (\mathrm{LY} 4) \sigma_{x, y, z}[x * y, z, u]=0, \\
& (\mathrm{LY} 5)[x, y, u * v]=[x, y, u] * v+u *[x, y, v], \\
& \text { (LY6) }[u, v,[x, y, z]]=[[u, v, x], y, z]+[x,[u, v, y], z]+ \\
& \quad[x, y,[u, v, z]],
\end{aligned}
$$

for all $u, v, x, y, z$, in $T$, where $\sigma_{x, y, z}$ denotes the sum over cyclic permutation of $x, y, z$. The bilinear map $*$ sometimes will be denoted by juxtaposition. If $x * y=0, \forall x, y \in T$, one gets a Lie triple system $(T,[\cdot, \cdot \cdot \cdot])$, while $[x, y, z]=0$ in $(T, *,[\cdot, \cdot, \cdot])$ induces a Lie algebra $(T, *)$.

Lie-Yamaguti algebras were introduced by Yamaguti [1] (who formerly called them "generalized Lie triple systems") in an algebraic study of the characteristic properties of the torsion and curvature of a homogeneous space with canonical connection [2]. Later on, these algebraic objects were called "Lie triple algebras" [3] and the terminology of "Lie-Yamaguti algebras" is introduced in [4] for these algebras. For further development of the theory of Lie-Yamaguti algebras one may refer, for example, to [5-8]. From the standard enveloping Lie algebra of a given Lie-Yamaguti algebra, the notions of the Killing-Ricci form and the invariant form of a Lie-Yamaguti algebra are introduced and studied in [9]. Further properties of invariant forms of Lie-Yamaguti algebras were considered in $[10]$.

Lie superalgebras as a $\mathbb{Z}_{2}$-graded generalization of Lie algebras are considered in $[11,12]$ while a $\mathbb{Z}_{2}$-graded generalization of Lie triple systems (called Lie supertriple systems) was first considered in [13]. For an application of Lie supertriple systems in physics, one may refer to [14]. Next, LieYamaguti superalgebras as a $\mathbb{Z}_{2}$-graded generalization of LieYamaguti algebras were first considered in [15].

Definition 1 (see [16]). A Lie-Yamaguti superalgebra is a $\mathbb{Z}_{2^{-}}$ graded vector space $T=T_{0} \oplus T_{1}$ with a binary operation denoted by juxtaposition satisfying $T_{i} T_{j} \subseteq T_{i+j}$ and a ternary operation $[\cdot, \cdot, \cdot]$ satisfying $\left[T_{i}, T_{j}, T_{k}\right] \subseteq T_{i+j+k}\left(i, j, k \in \mathbb{Z}_{2}\right)$ such that
(LYS1) $x y=-(-1)^{\bar{x} \bar{y}} y x$,
(LYS2) $[x, y, z]=-(-1)^{\bar{x} \bar{y}}[y, x, z]$,
$\left(\right.$ LYS3) $\circlearrowleft_{x, y, z}(-1)^{\bar{x} \bar{z}}\{((x y) z)+[x, y, z]\}=0$,
(LYS4) $\circlearrowleft_{x, y, z}(-1)^{\bar{x} \bar{z}}[x y, z, u]=0$,
(LYS5) $[x, y, u v]=[x, y, u] v+(-1)^{\bar{u}(\bar{x}+\bar{y})} u[x, y, v]$, 
(LYS6) $[u, v,[x, y, z]]=[[u, v, x], y, z]+(-1)^{\bar{x}(\bar{u}+\bar{v})}[x,[u, v$, $y], z]+(-1)^{(\bar{x}+\bar{y})(\bar{u}+\bar{v})}[x, y,[u, v, z]]$,

for all $u, v, x, y, z$, in $T$.

Observe that $T_{0}$ is a Lie-Yamaguti algebra.

As a part of the general theory of superalgebras, the notion of the Killing form of Lie algebras is extended to the one of Lie triple systems (see [17] and references therein), Lie superalgebras [12], and next Lie supertriple systems [18] (see also [19]).

In this paper we define and study the Killing form and invariant form of Lie-Yamaguti superalgebras as a generalization of the ones of both of Lie-Yamaguti algebras [9] and Lie supertriple systems $[18,19]$ including Lie superalgebras [12].

The paper is organized as follows. In Section 2 we record some useful results on Lie-Yamaguti superalgebras (see [16]). In Section 3 the Killing form of a Lie-Yamaguti superalgebra is defined (see Theorem 10 and Definition 11) and some of its properties are investigated (Proposition 13, Theorem 14, and Corollary 15). In Section 4 the invariant form of a LieYamaguti superalgebra is defined (Definition 16) and, under some conditions, it is shown (Theorem 21) that the Killing form of a Lie-Yamaguti superalgebra $T$ is nondegenerate if and only if the standard enveloping Lie superalgebra of $T$ is semisimple.

All vector spaces and algebras are finite-dimensional over a fixed ground field $\mathbb{K}$ of characteristic 0 .

\section{Some Basics on Lie-Yamaguti Superalgebras}

We give here some definitions and results which can be found in $[11,12,16]$.

A superalgebra over $\mathbb{K}$ is a $\mathbb{Z}_{2}$-graded algebra $A=A_{0} \oplus$ $A_{1}$, where $A_{i} A_{j} \subseteq A_{i+j},\left(i, j \in \mathbb{Z}_{2}\right)$. The subspaces $A_{0}$ and $A_{1}$ are called the even and the odd parts of the superalgebra and so are called the elements from $A_{0}$ and from $A_{1}$, respectively. Below, all the elements are assumed to be homogeneous, that is, either even or odd, and for a homogeneous element $x \in A_{i}$, $i=0,1$, the notation $\bar{x}=i$ is used and means the parity of $x$.

Let $G$ be the Grassmann algebra over $\mathbb{K}$ generated by the elements $1, e_{1}, \ldots, e_{n}$ such that $e_{i}^{2}=0, e_{i} e_{j}=-e_{j} e_{i}$ for $i \neq j$. The elements $1, e_{i_{1}} e_{i_{2}} \cdots e_{i_{r}}, i_{1}<i_{2}<\cdots<i_{r}$ form a basis of $G$. Denote by $G_{0}$ (resp., $G_{1}$ ) the span of the products of even length (resp., odd length) in the generators. The product of zero $e_{i}$ 's is by convention equal to 1 . Then $G=G_{0} \oplus G_{1}$ is an associative and supercommutative superalgebra; that is, $g_{1} g_{2}=(-1)^{\bar{g}_{1} \bar{g}_{2}} g_{2} g_{1}$, where $g_{1}, g_{2} \in G_{0} \cup G_{1}$. Let $A=A_{0} \oplus A_{1}$ be a superalgebra. Consider the graded tensor product $G \otimes A$ which becomes a superalgebra with the product given by $(x \otimes$ $\left.g_{1}\right)\left(y \otimes g_{2}\right)=(-1)^{\bar{x} \bar{g}_{2}} x y \otimes g_{1} g_{2}$, for homogeneous elements $g_{1}, g_{2} \in G, x, y \in A$ and grading given by $(G \otimes A)_{0}=G_{0} \otimes$ $A_{0} \oplus G_{1} \otimes A_{1},(G \otimes A)_{1}=G_{0} \otimes A_{1} \oplus G_{1} \otimes A_{0}$. The subalgebra $G(A)=(G \otimes A)_{0}=G_{0} \otimes A_{0} \oplus G_{1} \otimes A_{1}$ is called the Grassmann envelope of the superalgebra $A$.

Having in mind that if $V$ is a homogeneous variety of algebras [20], a superalgebra $A=A_{0} \oplus A_{1}$ is called a $V$ superalgebra, if its Grassmann envelope $G(A)$ belongs to $V$, we can state the following proposition.
Proposition 2. A superalgebra $T=T_{0} \oplus T_{1}$ equipped with bilinear and trilinear products verifying $T_{i} T_{j} \subseteq T_{i+j}$ and $\left[T_{i}, T_{j}, T_{k}\right] \subseteq T_{i+j+k}$ is a Lie-Yamaguti superalgebra if its Grassmann envelope $G(T)=G_{0} \otimes T_{0} \oplus G_{1} \otimes T_{1}$ is a Lie-Yamaguti algebra under the following products:

$$
\begin{aligned}
(x & \left.\otimes g_{1}\right)\left(y \otimes g_{2}\right)=(-1)^{\bar{x} \bar{y}} x y \otimes g_{1} g_{2} \\
{[x} & \left.\otimes g_{1}, y \otimes g_{2}, z \otimes g_{3}\right] \\
\quad= & (-1)^{\bar{x} \bar{y}+\bar{y} \bar{z}+\bar{x} \bar{z}}[x, y, z] \otimes g_{1} g_{2} g_{3} .
\end{aligned}
$$

Proof. The proof is straightforward by using the fact that, for any element $x \otimes g$ in $G(T)$, we have $\bar{x}=\bar{g}$.

Example 3. (1) Lie superalgebras are Lie-Yamaguti superalgebras with $[x, y, z]=0$.

(2) If $x y=0$ for any $x, y \in T_{0} \cup T_{1}$ then (LYS2), (LYS3), and (LYS6) define a Lie supertriple system.

(3) Let $M=M_{0} \oplus M_{1}$ be a Malcev superalgebra; that is, for any $x, y, z, t$ in $T$,

$$
\begin{aligned}
x y= & -(-1)^{\bar{x} \bar{y}} y x ; \\
-(-1)^{\bar{y} \bar{z}}(x z)(y t)= & ((x y) z) t \\
& +(-1)^{\bar{x}(\bar{y}+\bar{z}+\bar{t})}((y z) t) x \\
& +(-1)^{(\bar{x}+\bar{y})(\bar{z}+\bar{t})}((z t) x) y \\
& +(-1)^{\bar{t}(\bar{x}+\bar{y}+\bar{z})}((t x) y) z .
\end{aligned}
$$

It is shown in [16] that $M$ becomes a Lie-Yamaguti superalgebra if we set $[x, y, z]=x(y z)-(-1)^{\bar{x}} \bar{y}(x z)+(x y) z$. Conversely, if on a Malcev superalgebra $(M, \cdot)$ we define a trilinear operation by $[x, y, z]=x \cdot y z-(-1)^{\bar{x}} \bar{y} y \cdot x z+x y \cdot z$ then $(M, \cdot,[\cdot, \cdot, \cdot])$ is a Lie-Yamaguti superalgebra.

Definition 4. Let $T=T_{0} \oplus T_{1}$ be a Lie-Yamaguti superalgebra. A graded subspace $H=H_{0} \oplus H_{1}$ of $T$ is a graded LieYamaguti subalgebra of $T$ if $H_{i} H_{j} \subseteq H_{i+j}$ and $\left[H_{i}, H_{j}, H_{k}\right] \subseteq$ $H_{i+j+k}$ for any $i, j, k \in \mathbb{Z}_{2}$.

Definition 5. A graded subalgebra of a Lie-Yamaguti superalgebra $T$ is an invariant graded subalgebra (resp., an ideal) of $T$ if $[T, T, H] \subseteq H$ (resp., $T H \subseteq H$ and $[T, H, T] \subseteq H$ ).

If $H$ is an ideal of $T$, it is an invariant graded subalgebra of $T$. Obviously the center $Z(T)$ of a Lie-Yamaguti superalgebra $T$ defined by $Z(T)=\{x \in T, x y=0$ and $[x, y, z]=$ $0, \forall y, z \in T\}$ is an ideal of $T$.

Definition 6. Let $T=T_{0} \oplus T_{1}$ and $T^{\prime}=T_{0}^{\prime} \oplus T_{1}^{\prime}$ be Lie-Yamaguti superalgebras. A linear map $f: T \rightarrow T^{\prime}$ is said to be of degree $r$ if $f\left(T_{i}\right) \subseteq T_{r+i}^{\prime}$ for all $r, i \in \mathbb{Z}_{2}$.

Definition 7. Let $T=T_{0} \oplus T_{1}$ and $T^{\prime}=T_{0}^{\prime} \oplus T_{1}^{\prime}$ be LieYamaguti superalgebras. A linear map $f: T \rightarrow T^{\prime}$ is called a homomorphism of Lie-Yamaguti superalgebras if 
(1) $f$ preserves the grading, that is, $f\left(T_{i}\right) \subseteq T_{i}^{\prime}, i \in \mathbb{Z}_{2}$;

(2) $f(x y)=f(x) f(y)$;

(3) $f([x, y, z])=[f(x), f(y), f(z)]$ for any $x, y, z \in T_{0} \cup$ $T_{1}$.

Recall [11] that if $V=V_{0} \oplus V_{1}$ is a $\mathbb{Z}_{2}$-graded vector space then, if we set $\operatorname{End}_{r}(V)=\left\{f \in \operatorname{End}(V) / f\left(V_{i}\right) \subseteq V_{r+i}\right\}$, we obtain an associative superalgebra $\operatorname{End}(V)=\operatorname{End}_{0}(V) \oplus$ $\operatorname{End}_{1}(V) ; \operatorname{End}_{r}(V)$ consists of the linear mappings of $V$ into itself which are homogeneous of degree $r$. The bracket $[f, g]=$ $f g-(-1)^{\bar{f} \bar{g}} g f$ makes $\operatorname{End}(V)$ into a Lie superalgebra which we denote by $l(V)$ or $l(m, n)$ where $m=\operatorname{dim} V_{0}$ and $n=$ $\operatorname{dim} V_{1}$. Let $e_{1}, \ldots, e_{m}, e_{m+1}, \ldots, e_{m+n}$ be a basis of $V$. In this basis the matrix of $a \in l(m, n)$ is expressed as $\left(\begin{array}{ll}\alpha & \beta \\ \gamma & \delta\end{array}\right), \alpha$ being an $(m \times m)-, \delta$ an $(n \times n)-, \beta$ an $(m \times n)$-, and $\gamma$ an $(n \times m)$ matrix. The matrices of even elements have the form $\left(\begin{array}{ll}\alpha & 0 \\ 0 & \delta\end{array}\right)$ and those of odd ones $\left(\begin{array}{cc}0 & \beta \\ \gamma & 0\end{array}\right)$. For $a=\left(\begin{array}{ll}\alpha & \beta \\ \gamma & \delta\end{array}\right)$, the supertrace of $a$ is defined by $\operatorname{str}(a)=\operatorname{tr} \alpha-\operatorname{tr} \delta$ and does not depend on the choice of a homogeneous basis. We have $\operatorname{str}([a, b])=0$ that is $\operatorname{str}(a b)=(-1)^{\bar{a} \bar{b}} \operatorname{str}(b a)$ and $\operatorname{str}\left(a b a^{-1}\right)=\operatorname{str}(b)$.

Definition 8. Let $T=T_{0} \oplus T_{1}$ be a Lie-Yamaguti superalgebra; $D \in \operatorname{End}_{r}(T)$ is a superderivation of $T$ if, for any $x, y, z \in$ $T_{0} \cup T_{1}$,

$$
\begin{aligned}
D(x y)= & D(x) y+(-1)^{r \bar{x}} x D(y) ; \\
D([x, y, z])= & {[D(x), y, z]+(-1)^{r \bar{x}}[x, D(y), z] } \\
& +(-1)^{r(\bar{x}+\bar{y})}[x, y, D(z)] .
\end{aligned}
$$

Let $D_{r}(T)$ consist of all the superderivations of degree $r$ and $D(T)=D_{0}(T) \oplus D_{1}(T)$. It is easy to check that $D(T)$ is a graded subalgebra of End $(T)$ called the Lie superalgebra of superderivations of $T$.

Let $T=T_{0} \oplus T_{1}$ be a Lie-Yamaguti superalgebra. For any $x, y \in T_{0} \cup T_{1}$, denote by $D_{x, y}$ the endomorphism of $T$ defined by $D_{x, y}(z)=[x, y, z]$ for any $z \in T$. We have, for any $x, y \in$ $T_{0} \cup T_{1}, r \in \mathbb{Z}_{2}, D_{x, y}\left(T_{r}\right) \subseteq T_{r+\bar{x}+\bar{y}}$; that is, $D_{x, y}$ is a linear map of degree $\bar{x}+\bar{y}$. Moreover, it comes from (LYS5) and (LYS6) that

$$
\begin{aligned}
D_{x, y}(z w)= & D_{x, y}(z) w+(-1)^{\bar{z}(\bar{x}+\bar{y})} z D_{x, y}(w) ; \\
D_{x, y}([z, v, w])= & {\left[D_{x, y}(z), v, w\right] } \\
& +(-1)^{\bar{z}(\bar{x}+\bar{y})}\left[z, D_{x, y}(v), w\right] \\
& +(-1)^{(\bar{z}+\bar{v})(\bar{x}+\bar{y})}\left[z, v, D_{x, y}(w)\right]
\end{aligned}
$$

for any $x, y, z, v, w \in T_{0} \cup T_{1}$. It follows that $D_{x, y}$ is a superderivation of $T$ called an inner superderivation of $T$.

Let $D(T, T)$ be the vector space spanned by all $D_{x, y}(x, y \in T)$.

We can define naturally a $\mathbb{Z}_{2}$-gradation by setting $D(T, T)=D_{0}(T, T) \oplus D_{1}(T, T)$, where $D_{r}(T, T)$ consists of the superderivation $D_{x, y}$ of degree $r$. From (5) we also have that, for any $x, y, z, v, w \in T_{0} \cup T_{1}$,

$$
\left[D_{x, y}, D_{z, v}\right]=D_{[x, y, z], v}+(-1)^{\bar{z}(\bar{x}+\bar{y})} D_{z,[x, y, v]} .
$$

It is clear from (6) that $D(T, T)$ is a $\mathbb{Z}_{2}$-graded Lie subalgebra of $D(T)$ called the Lie superalgebra of all inner superderivations of $T$.

Now, let $(T, \cdot,[\cdot, \cdot, \cdot])$ be a Lie-Yamaguti superalgebra.

Set $L_{i}(T)=T_{i} \oplus D_{i}(T, T), i=0,1$, and define a new bracket operation in $L(T)=L_{0}(T) \oplus L_{1}(T)=T \oplus D(T, T)$ as follows: for any $x, y \in T_{0} \cup T_{1}, D_{1}, D_{2} \in D_{0}(T, T) \cup D_{1}(T, T)$,

$$
\begin{aligned}
{[x, y] } & =x y+D_{x, y} ; \\
{[D, x] } & =-(-1)^{\bar{x} \bar{D}}[x, D]=D(x) ; \\
{\left[D_{1}, D_{2}\right] } & =D_{1} D_{2}-(-1)^{\bar{D}_{1} \bar{D}_{2}} D_{2} D_{1} .
\end{aligned}
$$

Theorem 9. Let $T=T_{0} \oplus T_{1}$ be a Lie-Yamaguti superalgebra. Then

(1) $L(T)$ is a Lie superalgebra called the standard enveloping Lie superalgebra of $T$ and $D(T, T)$ becomes a graded subalgebra of $L(T)$.

(2) If $H$ is an ideal of $T$ then $H \oplus D(T, H)$ is an ideal of $T \oplus D(T, T)$.

Proof. The bracket $[\cdot, \cdot]$ is bilinear by definition and $X Y=$ $-(-1)^{\bar{X}} \bar{Y} Y X$ for any $X, Y \in L(T)$ by (LYS1) and (LYS2). Jacobi's superidentity follows from (LTS3-6).

(2) is obvious.

\section{Killing Forms of Lie-Yamaguti Superalgebras}

The definition of the Killing form given here for Lie-Yamaguti superalgebras stems from [9] in the case of Lie-Yamaguti algebras and extends the one given in [18] for Lie supertriple systems. Let $T=T_{0} \oplus T_{1}$ be an $n$-dimensional Lie-Yamaguti superalgebra. Denote by $\alpha$ the Killing form of the standard enveloping Lie superalgebra $L(T)=\left(T_{0} \oplus D_{0}(T, T)\right) \oplus$ $\left(T_{1} \oplus D_{1}(T, T)\right)$. Consider the bilinear form $\beta$ of $T$ obtained by restricting $\alpha$ to $T \times T$. For any $x, y, z$ in $T$, define the endomorphisms $L_{x}$ and $R_{x, y}$ of the vector space $T$ by $L_{x}(y)=$ $x y$ and $R_{x, y}(z)=(-1)^{\bar{z}(\bar{x}+\bar{y})}[z, x, y]=(-1)^{\bar{z}(\bar{x}+\bar{y})} D_{z, x}(y)$. It is clear that $R_{x, y}$ is of degree $\bar{x}+\bar{y}$ and $\left[D_{z, t}, R_{x, y}\right]=R_{[z, t, x], y}+$ $(-1)^{(\bar{z}+\bar{t}) \bar{x}} R_{x,[z, t, y]}$.

Theorem 10. For $x, y \in T$, we have

$$
\beta(x, y)=\operatorname{str}\left(L_{x} L_{y}\right)+\operatorname{str}\left(R_{x, y}+(-1)^{\bar{x} \bar{y}} R_{y, x}\right) .
$$


Proof. Let $\left\{a_{i}\right\},\left\{b_{i}\right\},\left\{u_{i}\right\},\left\{v_{i}\right\}$ be bases of $T_{0}, T_{1}, D_{0}(T, T)$, $D_{1}(T, T)$, respectively. For these bases, we express the operations of $T$ and $D(T, T)$ as follows:

$$
\begin{aligned}
a_{i} a_{j} & =\sum_{l} S_{i j}^{l} a_{l} ; \quad a_{i} a_{j} \in T_{0}, \\
a_{i} b_{j} & =\sum_{l} T_{i j}^{l} b_{l} ; \quad a_{i} b_{j} \in T_{1}, \\
b_{i} b_{j} & =\sum_{l} R_{i j}^{l} a_{l} ; \quad b_{i} b_{j} \in T_{0}, \\
D_{a_{i}, a_{j}} & =\sum_{\alpha} D_{i j}^{\alpha} u_{\alpha} ; \quad D_{a_{i}, a_{j}} \in D_{0}(T, T), \\
D_{a_{i}, b_{j}} & =\sum_{\alpha} C_{i j}^{\alpha} v_{\alpha} ; \quad D_{a_{i}, b_{j}} \in D_{1}(T, T), \\
D_{b_{i}, b_{j}} & =\sum_{\alpha} X_{i j}^{\alpha} u_{\alpha} ; \quad D_{b_{i}, b_{j}} \in D_{0}(T, T), \\
{\left[u_{\alpha}, a_{i}\right] } & =u_{\alpha}\left(a_{i}\right)=\sum_{j} K_{\alpha i}^{j} a_{j} ; \\
{\left[v_{\alpha}, a_{i}\right] } & =v_{\alpha}\left(a_{i}\right)=\sum_{j} L_{\alpha i}^{j} b_{j} ; \\
{\left[u_{\alpha}, b_{i}\right] } & =u_{\alpha}\left(b_{i}\right)=\sum_{j} H_{\alpha i}^{j} b_{j} ; \\
{\left[v_{\alpha}, b_{i}\right] } & =v_{\alpha}\left(b_{i}\right)=\sum_{j} Q_{\alpha i}^{j} a_{j} .
\end{aligned}
$$

To prove the theorem, it suffices to show that $\beta\left(a_{i}, a_{j}\right)=$ $\alpha\left(a_{i}, a_{j}\right), \beta\left(a_{i}, b_{j}\right)=\alpha\left(a_{i}, b_{j}\right)$ and $\beta\left(b_{i}, b_{j}\right)=\alpha\left(b_{i}, b_{j}\right)$. Since $\left(L_{a_{i}} L_{b_{j}}\right)\left(T_{0}\right) \subseteq T_{1}$ and $\left(L_{a_{i}} L_{b_{j}}\right)\left(T_{1}\right) \subseteq T_{0}$, we have $\operatorname{str}\left(L_{a_{i}} L_{b_{j}}\right)=0$. Also, $R_{a_{i}, b_{j}}\left(T_{1}\right) \subseteq T_{0}$ and $R_{a_{i} b_{j}}\left(T_{0}\right) \subseteq T_{1}$ give $\operatorname{str}\left(R_{a_{i}, b_{j}}+R_{b_{j}, a_{i}}\right)=0$ and then $\beta\left(a_{i}, b_{j}\right)=0=\alpha\left(a_{i}, b_{j}\right)$ because of the consistency property of $\alpha\left(a_{i} \in T_{0} \oplus D_{0}(T, T), b_{j} \in T_{1} \oplus\right.$ $\left.D_{1}(T, T)\right)$. Hence, it remains to show that $\beta\left(a_{i}, a_{j}\right)=\alpha\left(a_{i}, a_{j}\right)$ and $\beta\left(b_{i}, b_{j}\right)=\alpha\left(b_{i}, b_{j}\right)$. The operations in $T$ and the identities (7) imply the following:

$$
\begin{aligned}
{\left[a_{i},\left[a_{j}, a_{k}\right]\right]=} & {\left[a_{i}, a_{j} a_{k}+D_{a_{j}, a_{k}}\right] } \\
= & {\left[a_{i}, \sum_{m} S_{j k}^{m} a_{m}+\sum_{\alpha} D_{j k}^{\alpha} u_{\alpha}\right] } \\
= & \sum_{m} S_{j k}^{m}\left(a_{i} a_{m}+D_{a_{i}, a_{m}}\right)-\sum_{\alpha} D_{j k}^{\alpha} \sum_{l} K_{\alpha i}^{l} a_{l} \\
= & \sum_{m} S_{j k}^{m}\left(\sum_{l} S_{i m}^{l} a_{l}+\sum_{\alpha} D_{i m}^{\alpha} u_{\alpha}\right) \\
& -\sum_{\alpha, l} D_{j k}^{\alpha} K_{\alpha i}^{l} a_{l} \\
= & \sum_{m, l} S_{j k}^{m} S_{i m}^{l} a_{l}+\sum_{m, \alpha} S_{j k}^{m} D_{i m}^{\alpha} u_{\alpha} \\
& -\sum_{\alpha, l} D_{j k}^{\alpha} K_{\alpha i}^{l} a_{l} .
\end{aligned}
$$

In a similar way, we get

$$
\begin{aligned}
{\left[a_{i},\left[a_{j}, b_{k}\right]\right]=} & \sum_{m, l} T_{j k}^{m} T_{i m}^{l} b_{l}+\sum_{m, \alpha} T_{j k}^{m} C_{i m}^{\alpha} v_{\alpha} \\
& -\sum_{\alpha, l} C_{j k}^{\alpha} L_{\alpha i}^{l} b_{l}, \\
{\left[a_{i},\left[a_{j}, u_{\alpha}\right]\right]=} & -\left[a_{i}, u_{\alpha}\left(a_{j}\right)\right]=-\left[a_{i}, \sum_{m} K_{\alpha j}^{m} a_{m}\right] \\
= & -\sum_{m, l} K_{\alpha j}^{m} S_{i m}^{l} a_{l}-\sum_{m, \beta} K_{\alpha j}^{m} D_{i m}^{\beta} u_{\beta}, \\
{\left[a_{i},\left[a_{j}, v_{\alpha}\right]\right]=} & -\left[a_{i}, v_{\alpha}\left(a_{j}\right)\right]=-\left[a_{i}, \sum_{m} L_{\alpha j}^{m} b_{m}\right] \\
= & -\sum_{m, l} L_{\alpha j}^{m} T_{i m}^{l} b_{l}-\sum_{m, \beta} L_{\alpha j}^{m} C_{i m}^{\beta} v_{\beta} .
\end{aligned}
$$

Also,

$$
\begin{aligned}
L_{a_{i}} L_{a_{j}}\left(a_{k}\right) & =a_{i}\left(a_{j} a_{k}\right)=a_{i}\left(\sum_{p} S_{j k}^{p} a_{p}\right) \\
& =\sum_{p} S_{j k}^{p}\left(a_{i} a_{p}\right)=\sum_{p, l} S_{j k}^{p} s_{i p}^{l} a_{l}, \\
L_{a_{i}} L_{a_{j}}\left(b_{k}\right) & =a_{i}\left(a_{j} b_{k}\right)=a_{i}\left(\sum_{p} T_{j k}^{p} b_{p}\right) \\
& =\sum_{p} T_{j k}^{p}\left(a_{i} b_{p}\right)=\sum_{p, l} T_{j k}^{p} T_{i p}^{l} b_{l}, \\
R_{a_{i}, a_{j}}\left(a_{k}\right) & =\left[a_{k}, a_{i}, a_{j}\right]=D_{a_{k}, a_{i}}\left(a_{j}\right)=\sum_{\alpha} D_{k i}^{\alpha} u_{\alpha}\left(a_{j}\right) \\
& =\sum_{\alpha} D_{k i}^{\alpha} \sum_{m} K_{\alpha j}^{m} a_{m}=\sum_{\alpha, m} D_{k i}^{\alpha} K_{\alpha j}^{m} a_{m} ; \\
R_{a_{i}, a_{j}}\left(b_{k}\right) & =\left[b_{k}, a_{i}, a_{j}\right]=D_{b_{k}, a_{i}}\left(a_{j}\right) \\
& =-\sum_{\alpha} C_{i k}^{\alpha} v_{\alpha}\left(a_{j}\right)=-\sum_{\alpha} C_{i k}^{\alpha} \sum_{m} L_{\alpha j}^{m} b_{m} \\
& =-\sum_{\alpha, m} C_{i k}^{\alpha} L_{\alpha j}^{m} b_{m} .
\end{aligned}
$$

By interchanging $i$ and $j$, we have

$$
\begin{aligned}
& R_{a_{j}, a_{i}}\left(a_{k}\right)=\sum_{\alpha, m} D_{k j}^{\alpha} K_{\alpha i}^{m} a_{m} ; \\
& R_{a_{j}, a_{i}}\left(b_{k}\right)=-\sum_{\alpha, m} C_{j k}^{\alpha} L_{\alpha i}^{m} b_{m} .
\end{aligned}
$$


Therefore,

$$
\begin{aligned}
& \beta\left(a_{i}, a_{j}\right)=\alpha\left(a_{i}, a_{j}\right) \\
& =\sum_{m, k} S_{j k}^{m} S_{i m}^{k}-\sum_{\alpha, k} D_{j k}^{\alpha} K_{\alpha i}^{k} a_{k}-\sum_{m, k} T_{j k}^{m} T_{i m}^{k}+\sum_{\alpha, k} C_{j k}^{\alpha} L_{\alpha i}^{k} \\
& \quad-\sum_{m, \alpha} K_{\alpha j}^{m} D_{i m}^{\alpha}+\sum_{m, \alpha} L_{\alpha j}^{m} C_{i m}^{\alpha}, \\
& \operatorname{str}\left(L_{a_{i}} L_{a_{j}}\right)+\operatorname{str}\left(R_{a_{j}, a_{i}}+R_{a_{j}, a_{i}}\right) \\
& =\sum_{p, k} S_{j k}^{p} S_{i p}^{k}-\sum_{p, k} T_{j k}^{p} T_{i p}^{k}+\sum_{\alpha, k} D_{k i}^{\alpha} K_{\alpha j}^{k}+\sum_{\alpha, k} D_{k j}^{\alpha} K_{\alpha i}^{k} \\
& \quad+\sum_{\alpha, k} C_{i k}^{\alpha} L_{\alpha j}^{k}+\sum_{\alpha, k} C_{j k}^{\alpha} L_{\alpha i}^{k}=\beta\left(a_{i}, a_{j}\right) .
\end{aligned}
$$

It remains to show that $\beta\left(b_{i}, b_{j}\right)=\operatorname{str}\left(L_{b_{i}} L_{b_{j}}\right)+\operatorname{str}\left(R_{b_{j}, b_{i}}+\right.$ $\left.R_{b_{j}, b_{i}}\right)$,

$$
\begin{aligned}
{\left[b_{i},\left[b_{j}, b_{k}\right]\right]=} & {\left[b_{i}, b_{j} b_{k}+D_{b_{j}, b_{k}}\right] } \\
= & {\left[b_{i}, \sum_{m} R_{j k}^{m} a_{m}+\sum_{\alpha} X_{j k}^{\alpha} u_{\alpha}\right] } \\
= & -\sum_{m, l} R_{j k}^{m} T_{m i}^{l} b_{l}-\sum_{m, \alpha} R_{j k m i}^{m \alpha} v_{\alpha} \\
& -\sum_{\alpha, l} X_{j k}^{\alpha} H_{\alpha i}^{l} b_{l} .
\end{aligned}
$$

Likewise, we have

$$
\begin{aligned}
{\left[b_{i},\left[b_{j}, a_{k}\right]\right]=} & -\sum_{m, l} T_{k j}^{m} R_{i m}^{l} a_{l}-\sum_{m, \alpha} T_{k j}^{m} X_{i m}^{\alpha} u_{\alpha} \\
& -\sum_{\alpha, l} C_{k j}^{\alpha} Q_{\alpha i}^{l} a_{l}, \\
{\left[b_{i},\left[b_{j}, u_{\alpha}\right]\right]=} & -\left[b_{i}, u_{\alpha}\left(b_{j}\right)\right]=-\left[b_{i}, \sum_{l} H_{\alpha j}^{l} b_{l}\right] \\
= & -\sum_{l} H_{\alpha j}^{l} R_{i l}^{m} a_{m}-\sum_{l, \beta} H_{\alpha j}^{l} X_{i l}^{\beta} u_{\beta}, \\
{\left[b_{i},\left[b_{j}, v_{\alpha}\right]\right]=} & {\left[b_{i}, v_{\alpha}\left(b_{j}\right)\right]=\left[b_{i}, \sum_{l} Q_{\alpha j}^{l} a_{l}\right] } \\
= & -\sum_{l, m} Q_{\alpha j}^{l} T_{l i}^{m} b_{m}-\sum_{l, \beta} Q_{\alpha j}^{l} C_{l i}^{\beta} v_{\beta} .
\end{aligned}
$$

Therefore,

$$
\begin{aligned}
\beta\left(a_{i}, a_{j}\right)= & \alpha\left(a_{i}, a_{j}\right) \\
= & -\sum_{m, k} T_{k j}^{m} R_{i m}^{k}-\sum_{\alpha, k} C_{k j}^{\alpha} Q_{\alpha i}^{k}+\sum_{m, k} R_{j k}^{m} T_{m i}^{k} \\
& +\sum_{\alpha, k} X_{j k}^{\alpha} H_{\alpha i}^{k}-\sum_{l, \alpha} H_{\alpha j}^{l} X_{i l}^{\alpha}+\sum_{l, \alpha} Q_{\alpha j}^{l} C_{l i}^{\alpha} .
\end{aligned}
$$

Now,

$$
\begin{aligned}
L_{b_{i}} L_{b_{j}}\left(a_{k}\right) & =b_{i}\left(b_{j} a_{k}\right)=-b_{i}\left(\sum_{m} T_{j k}^{m} b_{m}\right) \\
& =-\sum_{m} T_{j k}^{m}\left(b_{i} b_{m}\right)=-\sum_{m, l} T_{j k}^{m} R_{i m}^{l} a_{l} ; \\
L_{b_{i}} L_{b_{j}}\left(b_{k}\right) & =b_{i}\left(b_{j} b_{k}\right)=b_{i}\left(\sum_{m} R_{j k}^{m} a_{m}\right) \\
& =-\sum_{m} R_{j k}^{m}\left(a_{m} b_{i}\right)=-\sum_{m, l} R_{j k}^{m} T_{m i}^{l} b_{l} ; \\
R_{b_{i}, b_{j}}\left(a_{k}\right) & =\left[a_{k}, b_{i}, b_{j}\right]=D_{a_{k}, b_{i}}\left(b_{j}\right)=\sum_{\alpha} C_{k i}^{\alpha} v_{\alpha}\left(b_{j}\right) \\
& =\sum_{\alpha} C_{k i}^{\alpha} \sum_{l} Q_{\alpha j}^{l} a_{l}=\sum_{\alpha, l} C_{k i}^{\alpha} Q_{\alpha j}^{l} a_{l} ; \\
R_{b_{i}, b_{j}}\left(b_{k}\right) & =\left[b_{k}, b_{i}, b_{j}\right]=D_{b_{k}, b_{i}}\left(b_{j}\right)=\sum_{\alpha} X_{k i}^{\alpha} u_{\alpha}\left(b_{j}\right) \\
& =\sum_{\alpha} X_{k i}^{\alpha} \sum_{l} H_{\alpha j}^{l} b_{l}=\sum_{\alpha, l} X_{k i}^{\alpha} H_{\alpha j}^{l} b_{l} .
\end{aligned}
$$

By interchanging $i$ and $j$, we have

$$
\begin{aligned}
& R_{b_{j}, b_{i}}\left(a_{k}\right)=\sum_{\alpha, l} C_{k j}^{\alpha} Q_{\alpha i}^{l} a_{l} ; \\
& R_{b_{j}, b_{i}}\left(b_{k}\right)=\sum_{\alpha, l} X_{k j}^{\alpha} H_{\alpha i}^{l} b_{l}, \\
& \operatorname{str}\left(L_{b_{i}} L_{b_{j}}\right)+\operatorname{str}\left(R_{b_{j}, b_{i}}+R_{b_{j}, b_{i}}\right) \\
& =-\sum_{m, k} T_{j k}^{m} R_{i m}^{k}+\sum_{m, k} R_{j k}^{m} T_{m i}^{k}+\sum_{\alpha, k} C_{k i}^{\alpha} Q_{\alpha j}^{k}-\sum_{\alpha, k} X_{k i}^{\alpha} H_{\alpha j}^{k} \\
& \quad-\sum_{\alpha, k} C_{k j}^{\alpha} Q_{\alpha i}^{k}+\sum_{\alpha, k} X_{k j}^{\alpha} H_{\alpha i}^{k} b_{l}=\beta\left(b_{i}, b_{j}\right) .
\end{aligned}
$$

Hence the theorem is proved.

Definition 11. The bilinear form $\beta$ defined on the LieYamaguti superalgebra $T=T_{0} \oplus T_{1}$ by

$$
\beta(x, y)=\operatorname{str}\left(L_{x} L_{y}\right)+\operatorname{str}\left(R_{x, y}+(-1)^{\bar{x} \bar{y}} R_{y, x}\right)
$$

for $x, y \in T$ is called the Killing form of $T$.

Remark 12. Recall that if $T$ is a Lie superalgebra, then the Killing form $\beta$ on $T$ is defined as $\beta(x, y)=\operatorname{str}\left(L_{x} L_{y}\right)$, $x, y \in T$. Likewise, if $T$ is a Lie supertriple system (resp., a Lie-Yamaguti algebra), the Killing form on $T$ is defined as $\beta(x, y)=\operatorname{str}\left(R_{x, y}+(-1)^{\bar{x} \bar{y}} R_{y, x}\right)$ (resp., $\beta(x, y)=$ $\left.\operatorname{tr}\left(L_{x} L_{y}\right)+\operatorname{tr}\left(R_{x, y}+R_{y, x}\right)\right)$ with $L_{u}$ and $R_{u, v}$ defined according to the considered structure on $T$. So if a Lie-Yamaguti superalgebra $T$ is reduced to a Lie superalgebra (resp., a Lie supertriple system, a Lie-Yamaguti algebra), then $\beta$ as defined in Definition 11 is the Killing form of the Lie superalgebra (resp., the Lie supertriple system, the Lie-Yamaguti algebra) $T$. 
Proposition 13. Let $T=T_{0} \oplus T_{1}$ be a Lie-Yamaguti superalgebra with a Killing form denoted by $\beta$. Then,

(1) $\beta\left(T_{0}, T_{1}\right)=0$ (consistence),

(2) $\beta(x, y)=(-1)^{\bar{x} \bar{y}} \beta(y, x)$ (supersymmetry),

(3) $\beta(A(x), A(y))=\beta(x, y), A \in \operatorname{Aut}(T)$.

Proof. As $L_{T_{0}} L_{T_{1}}\left(T_{1}\right) \subseteq T_{0}, L_{T_{0}} L_{T_{1}}\left(T_{0}\right) \subseteq T_{1}, R_{T_{0}, T_{1}}\left(T_{0}\right) \subseteq T_{1}$ and $R_{T_{0}, T_{1}}\left(T_{1}\right) \subseteq T_{0}$ we can state that $\beta\left(T_{0}, T_{1}\right)=0$.

(2) comes from the definition of $\beta$.

Now, for any $A$ in $\operatorname{Aut}(T), x$ in $T, \overline{A(x)}=\bar{x}$, and

$$
\begin{aligned}
\beta(A(x), A(y)) \\
=\operatorname{str}\left(L_{A(x)} L_{A(y)}\right) \\
\quad+\operatorname{str}\left(R_{A(x), A(y)}+(-1)^{\bar{x} \bar{y}} R_{A(y), A(x)}\right) .
\end{aligned}
$$

As $A(x y)=A(x) A(y)$ then $A L_{x}(y)=L_{A(x)} A(y)$; that is, $A L_{x}=L_{A(x)} A$ and $A L_{x} A^{-1}=L_{A(x)}$.

Hence, $\operatorname{str}\left(L_{A(x)} L_{A(y)}\right)=\operatorname{str}\left(A L_{x} A^{-1} A L_{y} A^{-1}\right)=$ $\operatorname{str}\left(A L_{x} L_{y} A^{-1}\right)=\operatorname{str}\left(L_{x} L_{y}\right)$. Also, $A[x, y, z]=[A(x), A(y)$, $A(z)$ ] gives $A R_{y, z}(x)=R_{A(y), A(z)}(A(x))$; that is, $A R_{y, z} A^{-1}=$ $R_{A(y), A(z)}$. Then,

$$
\begin{aligned}
\beta(A(x), A(y)) \\
=\operatorname{str}\left(L_{A(x)} L_{A(y)}\right) \\
\quad+\operatorname{str}\left(R_{A(x), A(y)}+(-1)^{\bar{x} \bar{y}} R_{A(y), A(x)}\right) \\
=\operatorname{str}\left(L_{x} L_{y}\right) \\
\quad+\operatorname{str}\left(A R_{x, y} A^{-1}+(-1)^{\bar{x} \bar{y}} A R_{y, x} A^{-1}\right) \\
=\operatorname{str}\left(L_{x} L_{y}\right)+\operatorname{str}\left(A\left(R_{x, y}+(-1)^{\bar{x} \bar{y}} R_{y, x}\right) A^{-1}\right) \\
=\operatorname{str}\left(L_{x} L_{y}\right)+\operatorname{str}\left(R_{x, y}+(-1)^{\bar{x} \bar{y}} R_{y, x}\right) \\
=\beta(x, y) .
\end{aligned}
$$

Now, let $\gamma$ be a trilinear form in $T$ given by $\gamma(x, y, z)=$ $\operatorname{str}\left(D_{x, y} L_{z}\right)$ for any $x, y, z \in T$. We can easily see that, for any $x, y, z \in T, \gamma(x, y, z)=-(-1)^{\bar{x}} \bar{y} \gamma(y, x, z)$ and that $\gamma$ vanishes identically if $T$ is reduced to Lie superalgebra or Lie supertriple system.

Theorem 14. Let $T=T_{0} \oplus T_{1}$ be a Lie-Yamaguti superalgebra with a Killing form denoted by $\beta$. Then, $\beta$ satisfies the identities

$$
\begin{aligned}
& \beta(x y, z)+(-1)^{\bar{x} \bar{y}} \beta(y, x z) \\
& \quad=(-1)^{\bar{x} \bar{y}} \gamma(y, x, z)+(-1)^{(\bar{x}+\bar{y}) \bar{z}} \gamma(z, x, y) ; \\
& \beta(x,[y, z, w])+(-1)^{\bar{w}(\bar{y}+\bar{z})} \beta([x, w, y], z) \\
& \quad=(-1)^{\bar{w}(\bar{y}+\bar{z})} \gamma(x, w, y z)-(-1)^{\bar{x}(\bar{y}+\bar{z})} \gamma(y, z, x w)
\end{aligned}
$$

for all $x, y, z \in T$.
Proof. The Killing form $\alpha$ of $L=\left(T_{0} \oplus D_{0}(T, T)\right) \oplus\left(T_{1} \oplus\right.$ $\left.D_{1}(T, T)\right)$ satisfies $\alpha(y,[x, z])+(-1)^{\bar{x} y} \alpha([x, y], z)=0$; that is, $\alpha(y,[x, z])=\alpha([y, x], z)$. But, using $(7)$, we have

$$
\begin{aligned}
\alpha([x, y], z) & =\alpha\left(x y+D_{x, y}, z\right) \\
& =\alpha(x y, z)+\alpha\left(D_{x, y}, z\right) \\
& =\beta(x y, z)+\operatorname{str}\left(D_{x, y} L_{z}\right) \\
& =\beta(x y, z)+\gamma(x, y, z), \\
\alpha(y,[x, z]) & =\alpha\left(y, x z+D_{x, z}\right) \\
& =\alpha(y, x z)+\operatorname{str}\left(L_{y} D_{x, z}\right) \\
& =\beta(y, x z)+(-1)^{\bar{y}(\bar{x}+\bar{z})} \operatorname{str}\left(D_{x, z} L_{y}\right) \\
& =\beta(y, x z)+(-1)^{\bar{y}(\bar{x}+\bar{z})} \gamma(x, z, y) .
\end{aligned}
$$

Then the identity $\alpha(y,[x, z])+(-1)^{\bar{x}} \bar{y} \alpha([x, y], z)=0$ gives $\beta(x y, z)+\gamma(x, y, z)+(-1)^{\bar{x} \bar{y}} \beta(y, x z)+(-1)^{\bar{y} \bar{z}} \gamma(x, z, y)=$ 0 that is $\beta(x y, z)+(-1)^{\bar{x} \frac{y}{y}} \beta(y, x z)=-\gamma(x, y, z)-$ $(-1)^{\bar{y} \bar{z}} \gamma(x, z, y)=(-1)^{\bar{x} \bar{y}} \gamma(y, x, z)+(-1)^{(\bar{x}+\bar{y}) \bar{z}} \gamma(z, x, y)$ and (23) is obtained.

From $\alpha([y, x], z)=\alpha(y,[x, z])$ we deduce $\alpha(x,[w$, $[y, z]])=\alpha([x, w],[y, z])=\alpha([[x, w], y], z)$ that is $-(-1)^{\bar{w}(\bar{y}+\bar{z})} \alpha(x,[[y, z], w])=\alpha([[x, w], y], z)$ and

$$
\alpha(x,[[y, z], w])+(-1)^{\bar{w}(\bar{y}+\bar{z})} \alpha([[x, w], y], z)=0 .
$$

Then, using (7) again and developing (26), we have $\alpha(x,[y z+$ $\left.\left.D_{y, z}, w\right]\right)+(-1)^{\bar{w}(\bar{y}+\bar{z})} \alpha\left(\left[x w+D_{x, w}, y\right], z\right)=0$ and we get

$$
\begin{aligned}
& \alpha\left(x,(y z) w+D_{y z, w}+[y, z, w]\right) \\
& \quad+(-1)^{\bar{w}(\bar{y}+\bar{z})} \alpha\left((x w) y+D_{x w, y}+[x, w, y], z\right)
\end{aligned}
$$

$=0$.

This gives

$$
\begin{aligned}
& \beta(x,(y z) w)+\alpha\left(x, D_{y z, w}\right)+\beta(x,[y, z, w]) \\
& \quad+(-1)^{\bar{w}(\bar{y}+\bar{z})} \beta((x w) y, z) \\
& +(-1)^{\bar{w}(\bar{y}+\bar{z})} \alpha\left(D_{x w, y}, z\right) \\
& \quad+(-1)^{\bar{w}(\bar{y}+\bar{z})} \beta([x, w, y], z)=0, \\
& \beta(x,(y z) w)+\operatorname{str}\left(L_{x} L_{D_{y z, w}}\right)+\beta(x,[y, z, w]) \\
& \quad+(-1)^{\bar{w}(\bar{y}+\bar{z})} \beta((x w) y, z) \\
& \quad+(-1)^{\bar{w}(\bar{y}+\bar{z})} \operatorname{str}\left(L_{D_{x w, y}} L_{z}\right) \\
& \quad+(-1)^{\bar{w}(\bar{y}+\bar{z})} \beta([x, w, y], z)=0 .
\end{aligned}
$$


Thus,

$$
\begin{aligned}
& \beta(x,(y z) w)+(-1)^{\bar{x}(\bar{y}+\bar{z}+\bar{w})} \operatorname{str}\left(L_{D_{y z, w}} L_{x}\right) \\
& \quad+\beta(x,[y, z, w])+(-1)^{\bar{w}(\bar{y}+\bar{z})} \beta((x w) y, z) \\
& \quad+(-1)^{\bar{w}(\bar{y}+\bar{z})} \operatorname{str}\left(L_{D_{x w, y}} L_{z}\right) \\
& \quad+(-1)^{\bar{w}(\bar{y}+\bar{z})} \beta([x, w, y], z)=0
\end{aligned}
$$

that is,

$$
\begin{aligned}
& \beta(x,(y z) w)+(-1)^{\bar{x}(\bar{y}+\bar{z}+\bar{w})} \gamma(y z, w, x) \\
& \quad+\beta(x,[y, z, w])+(-1)^{\bar{w}(\bar{y}+\bar{z})} \beta((x w) y, z) \\
& +(-1)^{\bar{w}(\bar{y}+\bar{z})} \gamma(x w, y, z) \\
& \quad+(-1)^{\bar{w}(\bar{y}+\bar{z})} \beta([x, w, y], z)=0 .
\end{aligned}
$$

This implies

$$
\begin{aligned}
\beta(x,[y, z, w])+(-1)^{\bar{w}(\bar{y}+\bar{z})} \beta([x, w, y], z) \\
=-\beta(x,(y z) w)-(-1)^{\bar{w}(\bar{y}+\bar{z})} \beta((x w) y, z) \\
\quad-(-1)^{\bar{x}(\bar{y}+\bar{z}+\bar{w})} \gamma(y z, w, x) \\
-(-1)^{\bar{w}(\bar{y}+\bar{z})} \gamma(x w, y, z) .
\end{aligned}
$$

But (23) gives

$$
\begin{aligned}
& (-1)^{\bar{w} \bar{x}} \beta(x, w(y z))+\beta(w x, y z) \\
& =(-1)^{\bar{w} \bar{x}} \gamma(x, w, y z) \\
& \quad+(-1)^{(\bar{w}+\bar{x})(\bar{y}+\bar{z})} \gamma(y z, w, x), \\
& (-1)^{\bar{w} \bar{x}} \beta((x w) y, z)+\beta(w x, y z) \\
& =\gamma(w x, y, z)+(-1)^{(\bar{w}+\bar{x})(\bar{y}+\bar{z})+\bar{y} \bar{z}} \gamma(z, y, w x) .
\end{aligned}
$$

Then,

$$
\begin{aligned}
-\beta( & x, w(y z))-(-1)^{\bar{w}(\bar{y}+\bar{z})} \beta((x w) y, z) \\
= & (-1)^{\bar{w}(\bar{y}+\bar{z})} \gamma(x, w, y z) \\
& +(-1)^{\bar{x}(\bar{w}+\bar{y}+\bar{z})} \gamma(y z, w, x) \\
& -(-1)^{\bar{w}(\bar{x}+\bar{y}+\bar{z})} \gamma(w x, y, z) \\
& -(-1)^{\bar{x}(\bar{w}+\bar{y}+\bar{z})+\bar{y} \bar{z}} \gamma(z, y, w x) .
\end{aligned}
$$

Hence, $\beta(x,[y, z, w])+(-1)^{\bar{w}(\bar{y}+\bar{z})} \beta([x, w, y], z)=(-1)^{\bar{w}(\bar{y}+\bar{z})} \gamma(x$, $w, y z)-(-1)^{\bar{x}(\bar{y}+\bar{z})} \gamma(z, y, w x)$ and (24) is proved.
Corollary 15. Let $T=T_{0} \oplus T_{1}$ be a Lie-Yamaguti superalgebra with a Killing form denoted by $\beta$. Then, $\beta$ satisfies the following for $x, y, z \in T$ :

$$
\begin{gathered}
([y, z, x], w)+(-1)^{\bar{x}(\bar{y}+\bar{z})} \beta(x,[y, z, w])=0 ; \\
\beta\left(R_{w, y}(x), z\right)-(-1)^{(\bar{w}+\bar{y}) \bar{x}+\bar{w} \bar{y}} \beta\left(x, R_{y, w}(z)\right) \\
=(-1)^{\bar{x}(\bar{w}+\bar{y})} \gamma(x, w, y z) \\
\quad+(-1)^{\bar{w}(\bar{y}+\bar{z})+\bar{x} \bar{z}} \gamma(y, z, x w) .
\end{gathered}
$$

Proof. Using (24) we have

$$
\begin{aligned}
& \beta([y, z, x], w)(-1)^{(\bar{w}+\bar{x})(\bar{y}+\bar{z})} \beta([x, w, y], z) \\
&-(-1)^{(\bar{w}+\bar{x})(\bar{y}+\bar{z})} \gamma(x, w, y z) \\
&-(-1)^{\bar{w} \bar{x}} \gamma(y, z, w x) ; \\
&(-1)^{\bar{x}(\bar{y}+\bar{z})} \beta(x,[y, z, w]) \\
&=-(-1)^{(\bar{w}+\bar{x})(\bar{y}+\bar{z})} \beta([x, w, y], z) \\
& \quad+(-1)^{(\bar{w}+\bar{x})(\bar{y}+\bar{z})} \gamma(x, w, y z) \\
&+(-1)^{\bar{w} \bar{x}} \gamma(y, z, w x) .
\end{aligned}
$$

By adding memberwise (36) and (37) we obtain the identity (34).

Also, the identity (37) is equivalent to $-(-1)^{\bar{y} \bar{z}} \beta(x,[z, y$, $w])+(-1)^{\bar{w}(\bar{y}+\bar{z})} \beta([x, w, y], z)=(-1)^{\bar{w}(\bar{y}+\bar{z})} \gamma(x, w, y z)-$ $(-1)^{\bar{x}(\bar{y}+\bar{z})} \gamma(y, z, x w)$. Then, we obtain $(-1)^{\bar{w} \bar{y}+\bar{x}(\bar{w}+\bar{y})} \beta\left(R_{w, y}(x)\right.$, $z, z)-\beta\left(x, R_{y, w}(z)\right)=(-1)^{\bar{w} \bar{y}} \gamma(w, x, y z)-(-1)^{\bar{x}(\bar{y}+\bar{z})+\bar{w} \bar{z}} \gamma(y$, $z, x w)$ that is $\beta\left(R_{w, y}(x), z\right)-(-1)^{(\bar{w}+\bar{y}) \bar{x}+\bar{w} \bar{y}} \beta\left(x, R_{y, w}(z)\right)=$ $(-1)^{\bar{x}(\bar{w}+\bar{y})} \gamma(x, w, y z)+(-1)^{\bar{w}(\bar{y}+\bar{z})+\bar{x} \bar{z}} \gamma(y, z, x w)$ and the remaining assertion is proved.

\section{Invariant Forms of Lie-Yamaguti Superalgebras}

In this section we introduced the concept of invariant forms of Lie-Yamaguti superalgebras as generalizations of those of Lie superalgebras and Lie supertriple systems.

Definition 16. An invariant form $b$ of a Lie-Yamaguti superalgebra $T=T_{0} \oplus T_{1}$ is a supersymmetric bilinear form on $T$ satisfying the identities

$$
\begin{array}{r}
b(x y, z)+(-1)^{\bar{x} \bar{y}} b(y, x z)=0 ; \\
b([x, w, y], z)+(-1)^{\bar{w}(\bar{y}+\bar{z})} b(x,[y, z, w])=0
\end{array}
$$

for all $x, y, z, w$ in $T$.

Remark 17. (1) If $\gamma=0$, the Killing form of $T$ is an invariant form of $T$. 
(2) If $T$ is reduced to a Lie supertriple system (resp., a Lie superalgebra, a Lie-Yamaguti algebra), then $b$ is reduced to an invariant form of a Lie supertriple system [19] (resp., a Lie superalgebra [12], a Lie-Yamaguti algebra [10]).

Definition 18. Let $b$ be an invariant form of a Lie-Yamaguti superalgebra $T$ and $S$ a subset of $T$. The orthogonal $S^{\perp}$ of $S$ with respect to $b$ is defined by $S^{\perp}=\{x \in T, b(x, y)=0, \forall y \in$ $S\}$. The invariant form $b$ is nondegenerate if $T^{\perp}=\{0\}$.

Lemma 19. Let $b$ be an invariant form of a Lie-Yamaguti superalgebra $T$. Then, for any $x, y, z, w$ in $T$, we have

$$
b([x, y, w], z)+(-1)^{(\bar{x}+\bar{y}) \bar{z}} b(w,[x, y, z])=0 .
$$

Proof. By interchanging $y$ and $w$ in (39) we have

$$
b([x, y, w], z)+(-1)^{\bar{y}(\bar{w}+\bar{z})} b(x,[w, z, y])=0
$$

that is $(-1)^{(\bar{x}+\bar{y}) \bar{z}} b(z,[x, y, w])+(-1)^{\bar{y}(\bar{w}+\bar{z})+\bar{w} \bar{z}} b(x,[w, z, y])=$ 0 by supersymmetry. Also by switching $z$ and $w$ in (41), we obtain $b([x, y, z], \widetilde{w})+(-1)^{\bar{y}(\bar{w}+\bar{z})} b(x,[z, w, y])=0$ that is

$$
b([x, y, z], w)-(-1)^{\bar{y}(\bar{w}+\bar{z})+\bar{w} \bar{z}} b(x,[w, z, y])=0 .
$$

Thus adding (41) and (42) we get (40) whence the lemma.

Lemma 20. Let $b$ be an invariant form of a Lie-Yamaguti superalgebra $T$. Then,

(1) $(T+[T, T, T])^{\perp}=Z(T)$ ifb is nondegenerate;

(2) if $H$ is an ideal of $T$ then $H^{\perp}$ is an ideal of $T$. In particular, $T^{\perp}$ is an ideal of $T$.

Proof. Consider $x$ in $(T+[T, T, T])^{\perp}$. Then, for any $u, v, w \in$ $T$, we have $b(x, u v)=0$ and $b(x,[u, v, w])=0$. This implies, by (38) and (39), that $(-1)^{\bar{u} \bar{x}} b(u x, v)=0$ and $(-1)^{\bar{w}(\bar{u}+\bar{v})} b([x, w, u], v)=0$ that is $b(u x, v)=0$ and $b([x, w, u], v)=0$. As $b$ is nondegenerate, we get $u x=0$ and $[x, w, u]=0$ for any $u, v, w \in T$. This gives $x \in Z(T)$.

Conversely, if $x \in Z(T)$, we have, for any $u, v, u^{\prime}, v^{\prime}, w^{\prime} \in$ $T, b\left(x, u v+\left[u^{\prime}, v^{\prime}, w^{\prime}\right]\right)=b(x, u v)+b\left(x,\left[u^{\prime}, v^{\prime}, w^{\prime}\right]\right)=0$ and $x \in(T+[T, T, T])^{\perp}$ whence $(T+[T, T, T])^{\perp}=Z(T)$.

Now, suppose that $H$ is an ideal of $T$ that is $T H \subseteq H$ and $[T, H, T] \subseteq H$; then for any $x, y \in T, u \in H^{\perp}$, and $h \in H$, we have $b(x u, h)=-(-1)^{\bar{x} \bar{u}} b(u, x h)=0$ and $b([x, u, y], h)=$ $-(-1)^{\bar{x} \bar{u}} b([u, x, y], h)=(-1)^{\bar{x}(\bar{y}+\bar{h})+\bar{x} \bar{u}} b(u,[y, h, x])=0$. Then $T H^{\perp} \subseteq H^{\perp}$ and $\left[T, H^{\perp}, T\right] \subseteq H^{\perp}$ which proves (2).

We are now ready to prove the following theorem.

Theorem 21. Let $T=T_{0} \oplus T_{1}$ be a Lie-Yamaguti superalgebra with $\gamma=0$. Then the Killing form $\beta$ is nondegenerate if and only if the standard enveloping Lie superalgebra $L(T)=T \oplus D(T, T)$ is a semisimple Lie superalgebra.

Proof. Let $\alpha$ be the Killing form of the Lie superalgebra $L(T)$. If $\gamma=0$, we have, for any $x, y, z \in T, \gamma(x, y, z)=$ $\operatorname{str}\left(D_{x, y} L_{z}\right)=0$ and

$$
\alpha\left(D_{x, y}, z\right)=0
$$

Then, using the invariance of $\alpha$ and (43), we have, for any $x, y, z, w \in T: \alpha\left([x, y], D_{z, w}\right)=\alpha\left(x,\left[y, D_{z, w}\right]\right)$; that is, by (7),

$$
\alpha\left(x y+D_{x, y}, D_{z, w}\right)=-(-1)^{\bar{y}(\bar{z}+\bar{w})} \alpha(x,[z, w, y]) \text { and }
$$
$\alpha\left(D_{x, y}, D_{z, w}\right)=-(-1)^{\bar{y}(\bar{z}+\bar{w})} \alpha(x,[z, w, y])$. This gives

$$
\alpha\left(D_{x, y}, D_{z, w}\right)=-(-1)^{\bar{y}(\bar{z}+\bar{w})} \beta(x,[z, w, y]) .
$$

Thus, if $\beta$ is nondegenerate, the restriction of $\alpha$ on $D(T, T) \times$ $D(T, T)$ is nondegenerate and $\alpha$ is nondegenerate.

Now, suppose that $\beta$ is degenerate. Then by the lemma above, $T^{\perp}$ is an ideal of $T$ so $T^{\perp} \oplus D\left(T, T^{\perp}\right)$ is a nonzero ideal of $T$.

Using the identities (43) and (44) we get

$$
\begin{aligned}
\alpha\left(T^{\perp} \oplus D\left(T, T^{\perp}\right), T \oplus D(T, T)\right) \\
=\alpha\left(T^{\perp}, T\right)+\alpha\left(T^{\perp}, D(T, T)\right)+\alpha\left(D\left(T, T^{\perp}\right), T\right) \\
\quad+\alpha\left(D\left(T, T^{\perp}\right), D(T, T)\right) \\
=\alpha\left(T^{\perp}, T\right)+\beta\left(T,\left[T, T^{\perp}, T\right]\right)=0 .
\end{aligned}
$$

It comes that $\alpha$ is degenerate and $T \oplus D(T, T)$ is not semisimple which proves the theorem.

The results of this paper could be used for a study of the structure of a pair consisting of a semisimple Lie superalgebra and its semisimple graded subalgebra.

\section{Competing Interests}

The authors declare that they have no competing interests.

\section{References}

[1] K. Yamaguti, "On the Lie triple system and its generalization," Journal of Science of the Hiroshima University, Series A, vol. 21, pp. 107-113, 1957/1958.

[2] K. Nomizu, "Invariant affine connections on homogeneous spaces," American Journal of Mathematics, vol. 76, pp. 33-65, 1954.

[3] M. Kikkawa, "Geometry of homogeneous Lie loops," Hiroshima Mathematical Journal, vol. 5, no. 2, pp. 141-179, 1975.

[4] M. K. Kinyon and A. Weinstein, "Leibniz algebras, Courant algebroids, and multiplications on reductive homogeneous spaces," American Journal of Mathematics, vol. 123, no. 3, pp. 525-550, 2001.

[5] P. Benito, A. Elduque, and F. Martín-Herce, "Irreducible LieYamaguti algebras," Journal of Pure and Applied Algebra, vol. 213, no. 5, pp. 795-808, 2009.

[6] P. Benito, A. Elduque, and F. Martín-Herce, "Irreducible LieYamaguti algebras of generic type," Journal of Pure and Applied Algebra, vol. 215, no. 2, pp. 108-130, 2011.

[7] J. Lin, L. Y. Chen, and Y. Ma, "On the deformation of LieYamaguti algebras," Acta Mathematica Sinica (English Series), vol. 31, no. 6, pp. 938-946, 2015.

[8] T. Zhang and J. Li, "Deformations and extensions of LieYamaguti algebras," Linear and Multilinear Algebra, vol. 63, no. 11, pp. 2212-2231, 2015. 
[9] M. Kikkawa, "On Killing-Ricci forms of Lie triple algebras," Pacific Journal of Mathematics, vol. 96, no. 1, pp. 153-161, 1981.

[10] M. Kikkawa, "Remarks on invariant forms of Lie triple algebras," Memoirs of the Faculty of Science and Engineering, Shimane University, vol. 16, pp. 23-27, 1982.

[11] V. G. Kac, "Lie superalgebras", Advances in Mathematics, vol. 26, no. 1, pp. 8-96, 1977.

[12] M. Scheunert, The Theory of Lie Superalgebras, Springer, Berlin, Germany, 1979.

[13] H. Tilgner, "A graded generalization of Lie triples," Journal of Algebra, vol. 47, no. 1, pp. 190-196, 1977.

[14] S. Okubo, "Parastatistics as Lie-supertriple systems," Journal of Mathematical Physics, vol. 35, no. 6, pp. 2785-2803, 1994.

[15] M. F. Ouédraogo, Sur les Superalgèbres triples de Lie, Thèse de Doctorat 3è cycle Mathématiques [Ph.D. thesis], Université de Ouagadougou, Ouagadougou, Burkina Faso, 1999.

[16] P. L. Zoungrana, "A note on Lie-Yamaguti superalgebras," Far East Journal of Mathematical Sciences, vol. 100, no. 1, pp. 1-18, 2016.

[17] T. S. Ravisankar, "Some remarks on Lie triple systems," Kumamoto Journal of Science (Mathematics), vol. 11, pp. 1-8, 1974.

[18] S. Okubo and N. Kamiya, "Quasi-classical Lie superalgebras and Lie supertriple systems," Communications in Algebra, vol. 30, no. 8, pp. 3825-3850, 2002.

[19] Z. Zhixue and J. Peipei, “The Killing forms and decomposition theorems of Lie supertriple systems," Acta Mathematica Scientia. Series B, vol. 29, no. 2, pp. 360-370, 2009.

[20] I. P. Shestakov, "Prime Mal'tsev superalgebras," Matematicheskii Sbornik, vol. 182, no. 9, pp. 1357-1366, 1991 (Russian). 


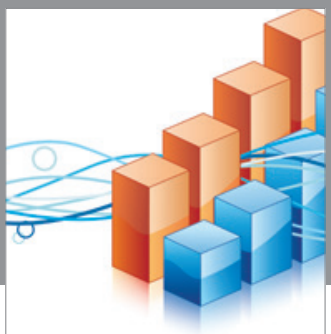

Advances in

Operations Research

vatem alat4

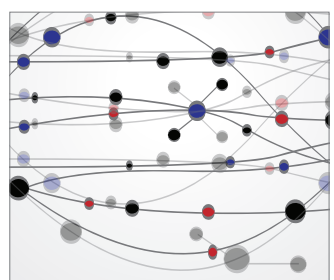

\section{The Scientific} World Journal
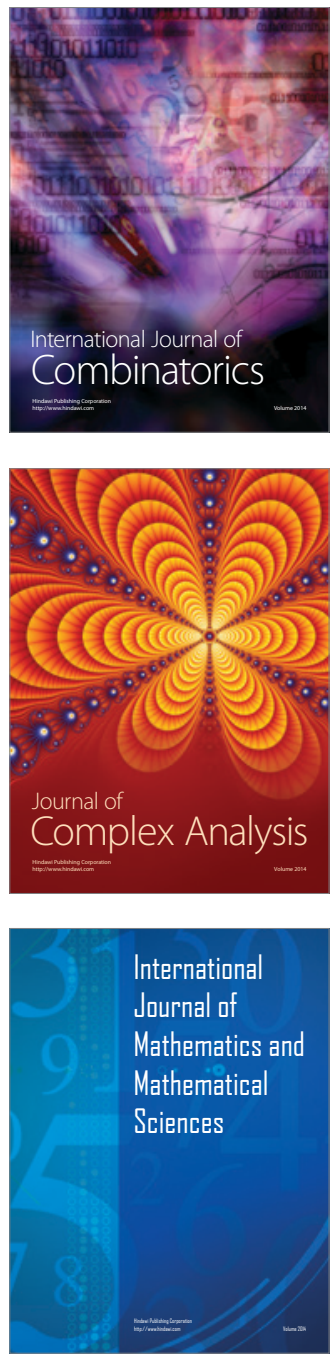
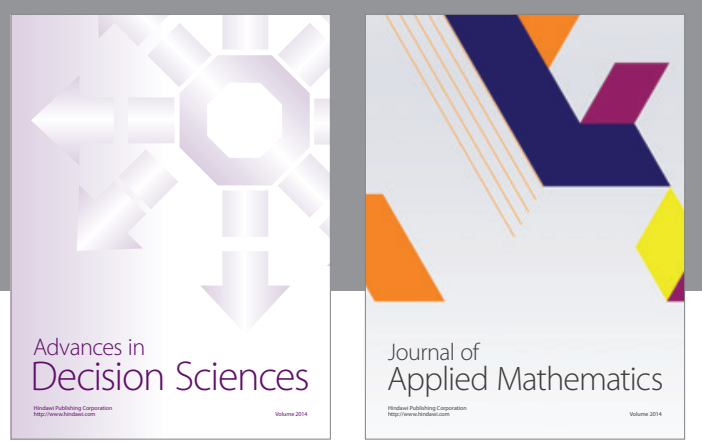

Algebra

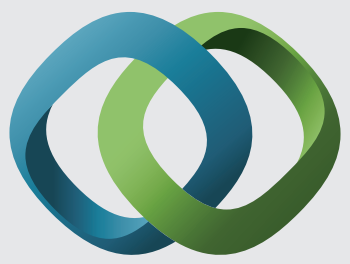

\section{Hindawi}

Submit your manuscripts at

https://www.hindawi.com
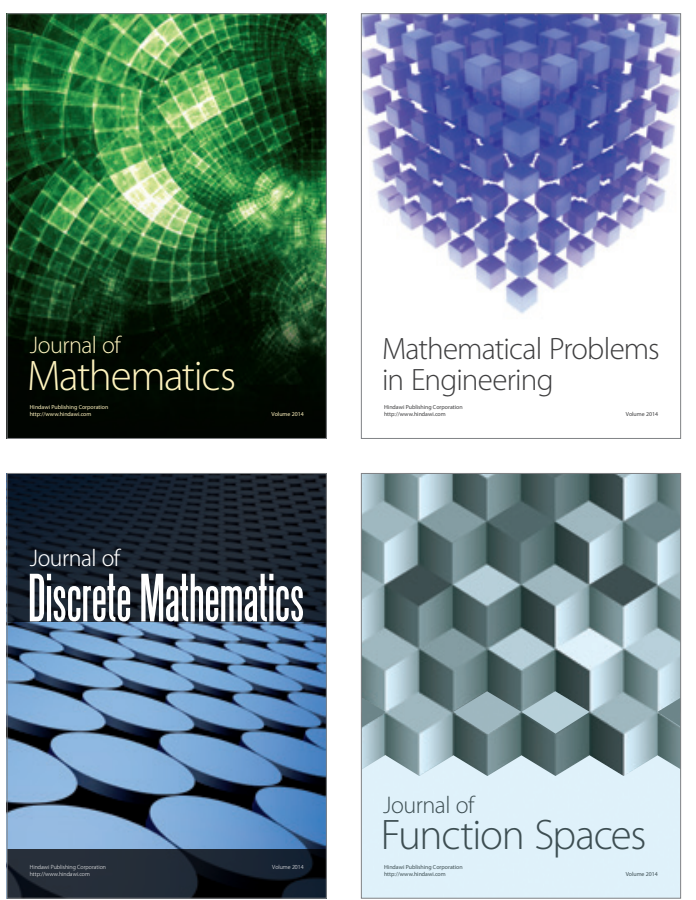

Mathematical Problems in Engineering
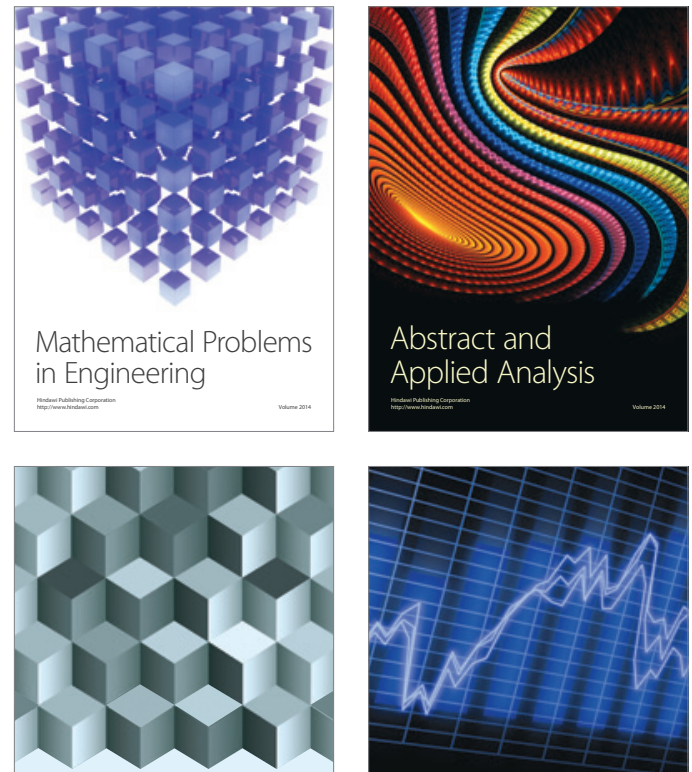

Journal of

Function Spaces

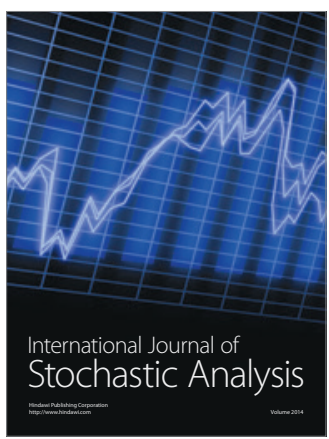

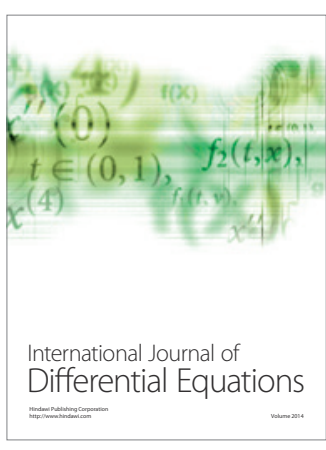
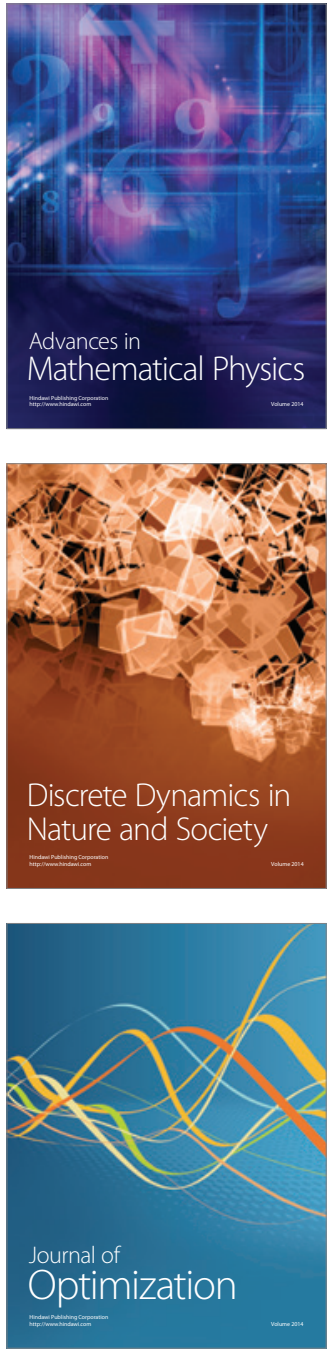